\title{
L'UBIQUITE RESIDENTIELLE AFRICAINE ET LE CONCEPT DES MIGRATIONS
}

Par

LUTUTALA Mumpasi

\author{
Département de Démographie \\ Université de Kinshasa \\ (Zaire)
}

\section{INTRODUCTION}

Le concept des migrations fait 1 'objet depuis un certain temps d'un débat qui promet d'être fructueux pour assurer une meilleure compréhension de ce phénomène. Ce débat trouve son fondement dans 1 'intérêt de plus en plus grand pour les migrations et fait suite aux écueils méthodologiques auxquels on est confronté lors de leur étude.

Les écueils méthodologiques qui bloquent 1 'avancement des recherches démographiques sur les migrations seraient de trois ordres (Termote, 1983:1):

1) la rareté et 1a non fiabilité des données.

2) la "mauvaise" conceptualisation du phénomène, et

3) les limites des méthodes d'analyse.

En fait, le problème prépondérant nous parait ètre celui de la "mauvaise" conceptualisation des migrations africaines. En effet, l'étude de celles-ci a été généralement conçue comme celle d'un facteur de la croissance (ou la décroissance) et de la distribution spatiale de la population. Dans cette optique, les approches classiques jusqu'ici mises en oeuvre sont plus ou moins adaptées, notamment la définition classique de la migration. Celle-ci paraît très restrictive lorsqu'on veut approfondir la connaissance des mécanismes des migrations: modalités et types de déplacement, rôle et place du phénomène dans le "régime démographique" (1). etc...

Des tentatives de redéfinition du concept de migration ont été faites par référence à des critères pertinents tels que "espace de vie" (Brunet, 1975 : Courgeau, 1980 ; Poulain, 1985), "aire d'action migratoire" (Dupont et Dureau, 1986) et plus récemment "résidence-base" (Domenach et Picouet, 1987). Cet article s'inscrit dans le cadre de ce questionnement et propose d'élargir, dans le contexte africain des migrations, la notion de résidence-base à celle d'espace de vie familial. Il $s$ 'appuie sur la force et la permanence d'une caractéristique fondamentale des sociétés africaines, à savoir le lien familial. Celui-ci explique les relations que le migrant entretient aussi 
bien dans son lieu d'accueil que dans son lieu d'origine et tous les autres lieux où résident les membres du clan. Il s'ensuit, pour ceux-ci, une "ubiquité résidentielle" (Dupont et Dureau, 1986) qui rend floue la dichotomie entre les déplacements temporaires et les déplacements définitifs.

Mais à la pertinence d'une nouvelle conceptualisation éventuelle des migrations africaines doivent être opposées les difficultés d'opérationnalisation. Cet article tente également de retracer ces difficultés et préconise, ou plutót avalise, une approche de type bibliographique relativement poussée qui permettrait de bâtir des typologies nouvelles des migrations africaines.

\section{De la définition classique des migrations}

On considère généralement les migrations comme "un mouvement des personnes traversant une certaine limite afin d'établir ailleurs une nouvelle résidence permanente" (Population Reference Bureau, 1986, dans Domenach et Picouet, 1987:469). L'espace et le temps sont donc deux dimensions intrinsèques des migrations.

Le critère "temps" rend compte d'une période d'absence au lieu d'origine habituelle (ou de présence au lieu de résidence actuelle). Cette période doit être suffisamment longue pour insinuer qu'il y a eu changement de lieu de résidence. Dans le cas de l'Afrique, où la grande majorité de la population exerce des activités agricoles, cette durée de résidence devrait être de six mois. En effet, si 1 'on tient compte des différentes phases du cycle de production agricole (abattage - ensemencement -sarclage - récolte), il parait difficile qu'une personne soit absente de son lieu de résidence habituelle (lieu de production et de reproduction) pendant plus de six mois. Une si longue absence traduirait un transfert de la résidence au nouveau lieu.

Quelques auteurs se sont déjà interrogés sur la pertinence de ce critère pour définir les migrations. Mentionnons à ce sujet la réflexion de Standing $(1984: 6)$. Pour 1ui, une des limites de ce critère est qu'il ne prend pas en consideration l'intention du migrant. On peut en effet se demander si une personne qui s'est installée à un endroit depuis moins de six mois, gênéralement considérée comme non-migrante, 1 'est vraiment si elle a une ferme intention de s'installer à cette nouvelle résidence (exemple : une personne nouvellement mariée). De méme, on doit se demander si une personne qui réside à un lieu depuis au moins six mois, et qui a toujours 1 'intention de retourner dans son lieu de résidence habituelle doit être considérée comme un migrant. D'autres cas méritent d'être soulignés, notamment celui des jeunes accouchées, des petites 
bonnes, des élèves à l'internat, des malades hospitalisés, etc... qui peuvent être "absents" de leur résidence pour plus de six mois.

Il semble que le critère "temps" ne soit pas non plus très considéré dans les statistiques officielles sur les migrations internationales. La distinction entre "visiteurs" et "immigrants" découle moins de la durée de résidence que de la finalité du séjour. Ainsi, une personne qui sêjourne pour des études dans certains pays est considérée comme "résident temporaire" même après sept années, tandis que celle qui est "reçue" comme "immigrant" est un "résident permanent" avant même, des fois, qu'elle ait fait son entrée dans le nouveau pays de résidence. C'est notamment le cas du Canada (Kelly, 1982).

Dans le cas de 1 'Afrique, on peut relever plusieurs limites d'une définition basée sur le critère "temps". Adepoju (1976:136) avait déjà montrê qu'au Nigêria, la "carrière migratoire" commençait et se terminait dans les milieux d'origine, et que très peu de migrants interrogês envisageaient de rester dans leurs villes de résidence de façon permanente. La plupart des migrants envisageaient retourner dans leurs villages d'origine aux ãges avancés. De même, les données de 1 'enquête sur les migrations que nous avons réalisèe à Kinshasa, capitale du Zaïre, auprès de 1853 ménages (2) montre que $30 \%$ des chefs de mênage avaient 1 'intention de retourner dans leurs villages d'origine.

\section{Tableau 1}

Répartition des chefs de ménage de sexe masculin selon leurs intentions migratoires, âges et durée de résidence (Kinshasa, 1984)

Ont 1 'intention de de retourner
Ages

N'ont pas 1 'intention de retourner

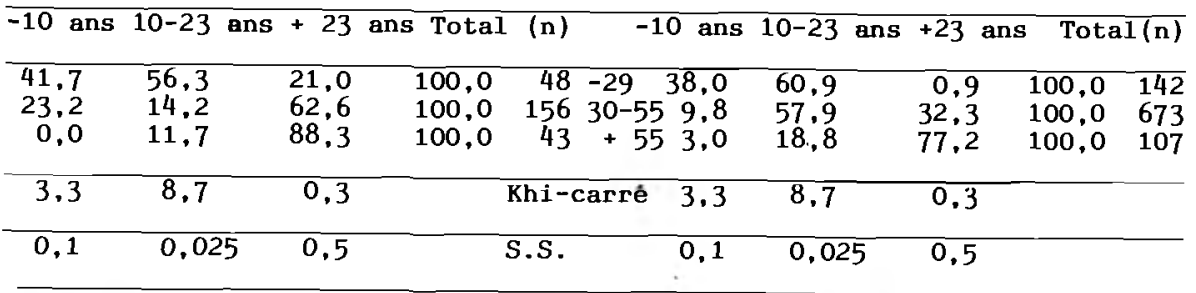

$(n)=$ effectifs enquêtés, les pourcentages sont calculés à partir des effectifs extrapolés

Source : Lututala, 1987, t.v.25 
Ce souci de retourner au village ne concerne pas seulement les migrants qui sont arrivés récemment dans la capitale, et qui considéreraient celle-ci comme un lieu de résidence provisoire. Au contraire, même les migrants ayant longtemps vécu à Kinshasa (+23 ans) envisagent retourner dans leurs villages d'origine.

De ce qui prêcède, on peut conclure que le critère "temps" ne peut, à lui seu1, permettre de mieux dêfinir et comptabiliser les migrations. Ce critère ne permet de rendre compte que d'une facette de la mobilitê humaine. Quant au critère "espace". 1a question qui se pose est celle de savoir ce qu'il faut entendre par "résidence" et ce qu'implique un changement de résidence.

\section{Les notions de "résidence" et d'"espace de vie" en migration}

La résidence d'un individu est souvent définie comme étant le lieu où il a coutume d'habiter (Dictionnaire démographique multilingue). En fait, le mot habiter réfère à un espace de vie, c'est-à-dire "I'ensemble des lieux fréquentés par un individu ou avec lesquels il est en rapport par 1 'intermédiaire des personnes s'y trouvant" (Poulain, 1985:7). C'est aussi "le cadre spatial le plus vaste à 1 'intérieur duquel s'effectuent la plupart des actes d'une population" (Brunet, 1975). Vue sous cet angle, la migration peut être définie comme un changement d'espace de vie (Brunet, 1975 ; Courgeau, 1980 et 1975).

Il semble cependant qu'en dépit de sa pertinence, le concept d'"espace de vie" se prèterait très mal à 1 'opérationnalisation : comment statistiquement rattacher le migrant à l'ensemble des lieux de son espace de vie? (Poulain, 1981:13). Cette impasse conduit généralement à 1 'abandon pur et simple de ce concept, au profit de celui plus restrictif et opératoire de résidence. Celle-ci est alors considérée comme étant le "centre de gravité" autour duquel gravitent les différents lieux de l'espace de vie (Poulain, 1985:8).

Si 1'observation de Poulain paraît pertinente, elle ne règle pas, nous semble-t-il, la question fondamentale de la signification sociale de la résidence, en Afrique comme ailleurs. En effet, $s^{\prime i l}$ est effectivement difficile de rattacher d'une façon "comptable" un individu aux différents lieux de son espace de vie, il est tout aussi difficile de concevoir la migration comme un changement de résidence. La notion de changement suppose une rupture, un mouvement irréversible, que de plus en plus d'auteurs mettent en cause. 
Dans un travail récent, Domenach et Picouet (1987) proposent un nouveau critère pour dêfinir les migrations. Il $s$ 'agit de la réversibilité de ce phénomène. D'après ces auteurs, la migration peut se définir en réfêrence à une résidence principale unique (résidencebase) ou non. Dans le premier cas, "le point de départ des déplacements reste le même, seule change leur destination" (p.475). Tout individu est donc rattaché à une résidence-base d'où partent et où aboutissent toutes ses migrations. Tout flux migratoire est donc rêversible. Cette réversibilité peut être de longue durée, renouvelable ou sporadique. La migration, on le voit, ne peut être conçue ici comme un changement définitif de rêsidence.

Dans le second cas, la migration peut se définir sans référence à une résidence-base, car celle-ci "n'intervient plus (à un moment donné) dans le système de reproduction familiale et socio-économique du groupe émigré" (ibidem, p.473). Ici, les flux migratoires sont irréversibles : et cette irréversibilité peut être forcée (cas des cataclysmes), provoquée (cas de surpopulation ou de besoin en force de travail) ou volontaire.

Les réflexions de Domenach et Picouet sur la résidence-base sont très pertinentes pour l'étude des migrations africaines. En effet, les sociétés africaines se caractérisent (encore) par une ubiquité résidentielle ou, en d'autres termes, le "caractère multispatial" de la vie (Dupont et Dureau, 1986:97). Ceci suppose deux choses : 1) 1'attachement a une résidence-base, et 2) 1a coexistence des membres familiaux.

\section{L'"ubiquité résidentielle" africaine et le contexte familial des migrations}

Le système de résidence de plusieurs sociètés africaines est une combinaison assez complexe des systèmes de résidence parentale et matrimoniale. Dans les systèmes matrilinéaires et virilocaux, les femmes mariées habitent à la résidence de leurs époux, tout en ètant attachées à 1 a rêsidence de leurs familles. Un tel système occasionne une circulation des femmes et des enfants entre la rêsidence de leur époux/père et celle de leurs familles. Le dêcès du conjoint/père donne lieu à un retour de 1a femme et des enfants "chez eux", dans la famille d'origine. Ils n'auront alors été que des migrants temporaires chez le conjoint/père, quelle que soit la durée du temps qu'ils y auront passé. Comme on le voit, l'Africain reste attaché à une résidence-base 
d'où partent et où aboutissent ces "migrations". Cette résidence-base $n$ 'est pas le logement, comme ce serait le cas en Occident (cfr Poulain, 1985:8), mais le "noyau familial" :

Si le lieu de naissance du migrant constitue un pôle de référence constant de son espace de vie, c'est qu'il demeure son lieu de reproduction sociale par excellence, la terre de ses ancêtres, où se dêroulent les cérémonies familiales et religieuses, où peuvent se conclure les mariages. C'est "au village" que sont les racines du migrant, et que réside le noyau fondateur et dirigeant de son groupe, méme si celui-ci est marqué par 1 'ubiquité de son système de fonctionnement (Dupont et Dureau, 1986:47).

Par ailleurs, 1'organisation sociale des sociétés africaines repose encore sur une coexistence des membres familiaux unis par des liens de consanguinité ou d'alliance. Comme 1'écrit Saïd (1969:39-43) dans le cas du Zaïre, ceux-ci demeurent tenus à la solidaritè et à 1a fusion de leurs consciences individuelles dans le "nous" collectif familial. Ceci explique les relations qui existent entre le migrant et les autres membres de famille, migrants et non-migrants, et nous permet de considérer, à l'instar de Arnold et al (1982), le migrant comme un membre extra-local de la famille. En tant que tel, il n'est pas tenu d'être physiquement présent dans sa rêsidence-base pour contribuer à la reproduction sociale de sa famille. Sa migration vers un lieu constitue alors un élargissement de son espace de vie ainsi que celui de toute la famille jusqu'à ce lieu. Ceci dit, par son appartenance à la famille, 1'Africain vit dans un espace qui s'étend jusqu'aux lieux où rêsident les autres membres de la famille. Et l'installation $d^{\prime}$ un membre dans un lieu quelconque donnerait lieu à une chaîne migratoire familiale, c'est-à-dire à de plus en plus d'arrivée d'autres membres de la famille, qui s'en vont résider "chez eux" (chez le membre déjà installé).

Plusieurs typologies migratoires peuvent ẽtre construites à partir de la notion de noyau familial. On pourrait par exemple considérer l'origine des migrants et distinguer les migrants qui arrivent au nouveau lieu de résidence directement de leur noyau familial, de ceux qui auront transité ailleurs. Une telle distinction ne serait pas vide de sens. En effet, on peut penser que les raisons ayant motivé chaque type de migrant diffèrent. Dans le cas du migrant qui part directement du noyau familial, on peut $s^{\prime}$ attendre à ce que ce soit plutôt la famille qui l'ait poussé à partir vendre sa force de travail, rejoindre ou chercher un conjoint, 
11.

poursuivre des études, etc... et contribuer ainsi à 1a reproduction familiale. Dans 1 'autre cas, d'autres raisons peuvent masquer cette pesanteur familiale: recherche d'un emploi plus rémunèrateur, commodités urbaines, etc...

Bien que les statistiques fassent défaut, on peut penser qu'au stade actuel de 1 'histoire des migrations africaines, la majorité des migrants proviennent encore directement de leur noyau familial, et les lieux où ils immigrent sont rarement des "milieux neutres". Il s'agirait au contraire d'endroits où se trouvent déjà installés d'anciens migrants de la famille. Les migrants s'orienteraient donc là où la famille a déjà transplanté une "branche" de son noyau familial.

Ceci permet d'envisager une autre typologie, axée sur la forme de participation des membres locaux et extra-locaux a la migration d'un membre de famille. On pourrait distinguer à ce sujet les migrants primaires des migrants secondaires. Nous entendons par migrants primaires ceux dont les familles d'appartenance n'ont aucun membre extra-local au lieu de destination au moment où ils entreprennent leur migration vers ce lieu. Quant aux migrants secondaires, il s'agit de ceux qui arrivent dans un lieu où résident déjà d'autres membres de famille. On peut distinguer parmi ceux-ci les migrants qui bénéficient d'un soutien de la part d'anciens migrants installés (migrants secondaires dêpendants) de ceux qui ne bénéficient d'aucun soutien (migrants secondaires indépendants).

Le tableau 2 ci-dessous montre que $13 \%$ seulement des chef's de ménage ont été les premiers migrants de leurs familles respectives à Kinshasa, alors que la grande majorité $(87 \%)$ sont arrivés dans 1a capitale après qu'un autre membre de leur famille s'y soit déjà installé. 
Tableau 2

Types de chefs de ménage migrants observés selon leurs ages actuels (Kinshasa, 1984)

Types

Ages $15-29 \quad 30-4 \overline{4} 45-5960$ \&+ Ensemble Effectifs

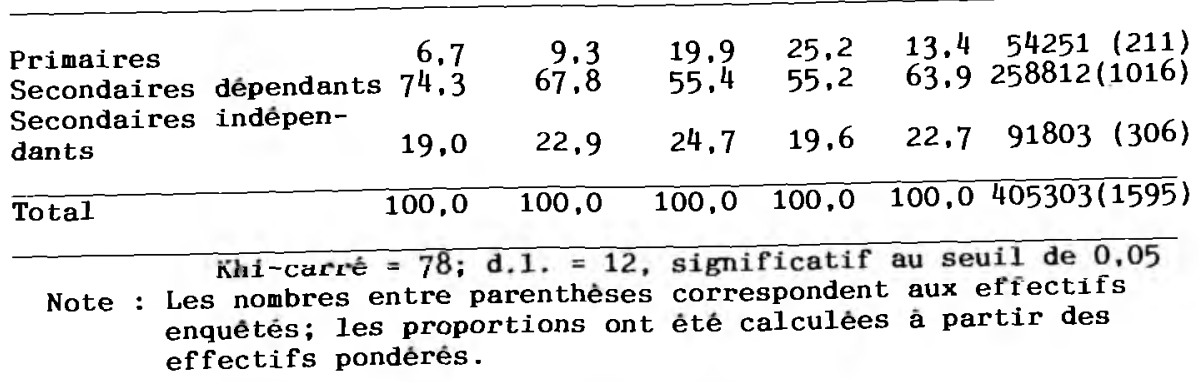

Source : Lututala (1987, t.V.27).

Comme on devait $s^{\prime} y$ attendre, la proportion des migrants primaires augmente avec 1 'âge, ou selon la durèe de résidence (car 1 'âge à la migration est presque le même pour toutes les générations). Ceci montre que 1 'organisation des stratégies migratoires au sein des familles est de plus en plus facilitée par 1 'existence des membres extra-locaux déjà installés à Kinshasa. Le tableau 3 qui suit montre que les migrants qui partent à Kinshasa sont presque tous accueillis par les membres de famille.

\section{Tableau 3}

Structure d'accueil des chefs de ménage migrants a Kinshasa

\begin{tabular}{|c|c|c|}
\hline & Effectifs & $\%$ \\
\hline $\begin{array}{l}\text { Conjoint } \\
\text { Enfant } \\
\text { Parents directs } \\
\text { Frées-neveux-cousins } \\
\text { Oncles-tantes } \\
\text { Gds-parents/Pts enfants } \\
\text { Parents du conjoint } \\
\text { Autres beaux-parents } \\
\text { Employeur-Internat } \\
\text { Amis/Amis des parents }\end{array}$ & $\begin{array}{rr}18 & 337 \\
2 & 085 \\
19 & 480 \\
158 & 879 \\
125 & 251 \\
5 & 994 \\
& 552 \\
21 & 852 \\
29 & 901 \\
22 & 385\end{array}$ & $\begin{array}{r}4,5 \\
0,5 \\
4,8 \\
39,3 \\
31,0 \\
1.5 \\
0,1 \\
5,4 \\
7,4 \\
5,5\end{array}$ \\
\hline
\end{tabular}


Le soutien apporté par les anciens migrants déjà installés a déjà étè mentionné dans plusieurs études sur les migrations africaines. Ce soutien consiste essentiellement à 1 'apprentissage d'un métier, la recherche d'emploi, le financement des études, 1'hẻbergement et 1a nourriture, etc...

Bien que l'ubiquitê rèsidentielle soit une réalité, il $n$ 'est pas facile d'en dèduire une typologie des migrations africaines. En effet, en dépit du fait que le noyau familial reste la résidence-base de tout africain, celui-ci n'y retourne pas nécessairement. c'est ce qui a été observê au Zaîre où il semble y avoir une ambivalence entre le désir des migrants de retourner dans leurs villages d'origine et 1 'avis contraire des membres locaux. Ceux-ci demandent au contraire aux migrants de demeurer à Kinshasa en vue de continuer à accueillir les nouveaux migrants, entre autres choses. Ils supportent, en revanche, les coûts pour la survie des membres qui sont à charge du migrant, mais qui $n$ 'ont pu ou pas encore voyagé avec lui. C'est ce que montre le tableau 4 ci-dessous.

\section{Tableau 4}

Sources de revenus des dépendants des chefs de ménage-migrants dans les milieux d'origine (Kinshasa, 1984)

\begin{tabular}{lll}
\hline Sources de revenus & Effectifs & $\%$ \\
\hline $\begin{array}{l}\text { Soutien des membres restés } \\
\text { au village }\end{array}$ & 24763 & 32.9 \\
$\begin{array}{l}\text { Dépendants eux-mèmes } \\
\text { Envois des fonds/biens }\end{array}$ & 20849 & 27.7 \\
du migrant & 16303 & 21,7 \\
Autres & 13312 & 17.7 \\
\hline Total & 75228 & 100,0 \\
\hline
\end{tabular}

Source : Lututala (1987:t.V.27)

Mais il a été observé aussi que les migrants euxmêmes posent certains préalables à leur retour. notamment 1 'amélioration des conditions de vie' au village. On voit ici que la réversibilitê peut être compromise par une êconomie en crise, comme c'est le cas en Afrique. Ceci permet de nuancer la proposition faite par Domenach et Picouet. Les auteurs $s^{\prime} y$ attendaient d'ailleurs car ils précisent que "les critères qui permettent d'apprécier la réversibilité éventuelle de $1 a$ 
migration (..) varient sensiblement selon les données du contexte socio-économique observe" (p.480).

\section{CONCLUSION}

Cet article se voulait une modeste contribution au débat actuel sur le concept des migrations. En proposant les concepts d'"espace de vie" et d' "aire d'action migratoire". les chercheurs occidentaux ont en effet contribué au souci de faire de l'espace une variable contextuelle dans 1'ètude des migrations (et non une constante comme c'est le cas en féconditê et mortalité; cfr Picouet, 1975). Aussi, s'il est établi que 1'individu reste bel et bien rattaché à une résidence-base par rapport à laquelle se comptabilisent ses migrations, il semble de plus en plus que cette résidence-base n'est pas nécessairement le logement. Elle ne serait pas non plus un espace (assez flou) de différents lieux qu'il fréquente. Elle serait plutôt, dans le contexte africain, un espace de vie familial, une sommation des résidences familiales qui gravitent, pour reprendre 1 'expression de Poulain, autour du noyau familial.

Il semble donc de plus en plus certain que les migrations, qu'elles soient africaines ou non, ne peuvent être définies correctement sans tenir compte du contexte socio-économique qui les sous-tend, ni non plus en se focalisant sur un seul discriminant. L'ubiquité résidentielle est, pensons-nous, une des spécificités africaines qu'il convient de prendre en considération. L'opérationnalisation de cette ubiquité ne peut évidemment être faite avec satisfaction dans 1'état actuel des statistiques. Les typologies que nous avons tenté de présenter n'ont pour seul but que d'illustrer la pertinence de cette caractéristique pour bâtir des typologies nouvelles des migrations africaines. Il s'agit d'une réflexion qui mérite d'être poursuivie. Les résultats des recensements et des enquétes démographiques devraient donc être complétés par une sêrie d'études longitudinales des migrations, à partir des monographies rétrospectives et des histoires migratoires de telle sorte qu'on dispose d'un peu plus de lumière sur les mécanismes de la migration, les liens entre cycles de vie d'un individu et ses migrations successives. Une telle étude n'est évidemment possible que moyennant une bonne définition de la rêsidence-base, laquelle se trouverait être, dans le cas de 1'Afrique, le noyau familial. 


\section{Notes}

* L'auteur remercie très sincèrement Philippe Antoine pour sa relecture-critique du manuscrit

(1) Sur le concept de "régime démographique", voir Cordell, Gregory et Piché (1987).

(2) Nous tenons à remercier le Centre de Recherches pour le Développement International (CRDI) qui a financé nos recherches doctorales, y compris la collecte et l'analyse des données de l'étude.

\section{BIBLIOGRAPHIE}

ADEPOJU, A. (1976) "Dynamics of Urban Population Growth in Nigeria : The Role of Repeated Migration", Jimlar Mutane, vol., n 1, $124-138$

ARNOLD, F et al (1982), "Shadow Households and Competing Auspices : Migration Behavior in the Philippines", paper, annual meeting of the P.A.A., April 28-May1

BRUNET, P. (1975) "Pour une nouvelle dêfinitition de la migration", in Migrations intérieures. Me thode d'observation et d'analyse, Paris, CNRS, 527-529

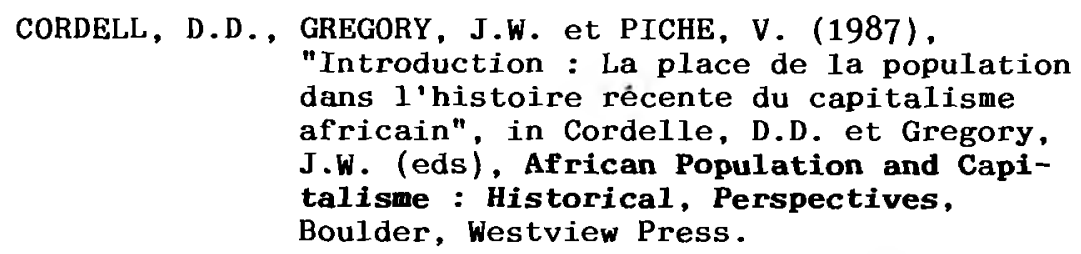

COURGEAU, D. (1975), "Mesure et analyse des migrations", in "L'analyse démographique et ses applications, Actes du collogue, Paris,CNRS, 151-161

COURGEAU, D. (1980), Analyse quantitative des migrations humaines, Paris, Masson, $220 \mathrm{p}$. 
DOMENACH, H. et PICOUET, M. (1987), "Le caractère de réversibilité dans 1 'étude de la migration". Population, n 3, mai-juin, 469-482

DUPONT, V. et DUREAU, F. (1986) Migration et dynamique des villes moyennes en Afrique de 1 'Ouest - le cas de 4 centres urbains en région de plantation (Côte d'Ivoire et Togo), Paris, Orstom, 155 p.

KELLY, J.J. (1982), "Possible Approaches to Improving the International Comparability of Immigration and Emigration Statistics", International Migration Review, vol. XVI n 3, 619-634

LUTUTALA, M. (1987), Dynamique des migrations au Zaire le réseau de Kinshasa, Thèse de $\mathrm{Ph}$.D. . Démographie, Universitè de Montréal, $427 \mathrm{p}$.

NATIONS UNIES (1983), "Les politiques de population en Afrique au début des annèes $80^{\prime \prime}$ in Actes du séminaire national sur les politiques de population au Mali, Bamako, 22-26 mars 96-124

NATIONS UNIES (1986) Examen et évaluation du plan d'action mondial sur la population, rapport 1984, New York, Etudes démographiques n 99 .

PICOUET, M. (1975), "Evolution et perspectives de 1a recherche démographique sur la migration" Cahiers ORSTOM, série Sc. Hum., vol. XII n4, 337-343

POULAIN, M. (1981), Contribution a l'analyse spatiale d'une matrice de migration interne, Louvain-1a-Neuve, Cabay, n 3, 325 p.

POULAIN, M. (1985), "La migration-concept et méthodes de mesure", in Chaire Quételet 1983:

Migration interne, collecte des données et méthodes d'analyse, Liège, Ordina, $7-38$ 
SAID, S.G. (1969), De Léopoldville à Kinshasa - 1a situation économique et financière du Congo-Belge au jour de 1 'indépendance, Bruxelles, CEPSI, 262 p.

STANDING, G. (1984), "Conceptualising Territorial Mobility", in Bilsborrow, R.E., Oberai, A.S. et Standing, G. (eds), Migration Surveys in Low Income Countries - Guideline for Survey Questionnaire Design, London and Sydney, Codom He1m, 31-59

TERmoTE, M. (1983), "La place de la migration dans la recherche démographique", Cahiers Québécois de Démographie, vol.12, n 2, 175-179

ZACHARIAH, K.C. , CLAIRIN, R. et CONDE, J . (1978), Aperçu sur les migrations en Afrique de 1 'Ouest (synthèse des travaux conjoints Banque Mondiale et OCDE). Paris. OCDE, CD/RI (78) 30, $87 \mathrm{p}$.

ZELINSKY, W. (1979), "The demographic transition : changing patterns of migration", in UIESP - La science de la population au service de 1 'homme - Population science in the service of making, Vienne. IUESP. 1965-188. 